\title{
A dual X-ray absorptiometry-based analysis of body fat distribution in healthy young adult women
}

\author{
P. Raftery, G. Boland, S. Leahy and P. Jakeman \\ Faculty of Education and Health Sciences, University of Limerick, Plassey Technological Park, Limerick, Republic \\ of Ireland
}

Previous research indicates that women with a greater proportion of abdominal fat tend to be more insulin resistant, hyperinsulinemic, glucose intolerant and dyslipidemic than women with a greater proportion of gluteal/femoral fat ${ }^{(1)}$. Accurate measurement of body fat and fat distribution is thus essential to assess the relation of regional fat mass to disease risk and change through dietary intervention; however, normative values for fat distribution do not exist. The UL Body Composition Study ${ }^{(2)}$ seeks to establish age- and gender-specific reference ranges for whole-body and segmental body composition based on a representative sample of the Irish population residing within the UL community. In this report, we analyse the relation between regional body fat and total body fat in healthy young adult women.

With ethical approval (ULREC 08/07) and informed written consent 115 young women aged 18-29 years, ranging from 15 to $47 \%$ body fat were recruited to the study. Total body fat mass and abdominal fat mass measured in the $\mathrm{L}_{1}-\mathrm{L}_{4}$ region was measured by dualenergy X-ray absorptiometry (iDXA ${ }^{\text {TM}}$; GE Healthcare, Chalfont St Giles, Bucks., UK; DXA) using the protocol outlined by Glickman et $\left.a l .{ }^{(3)}\right)$. This procedure of segmental, or region of interest, analysis has an inter-rater reliability of estimate of $<5 \%$.

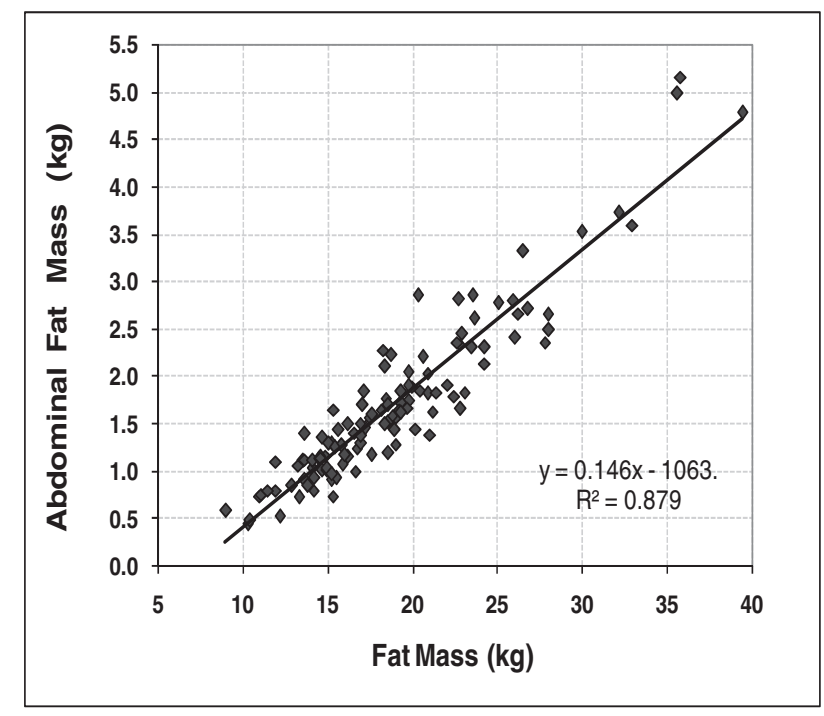

The subjects' average total fat mass 18.9 (sD, 5.6; min, 9.0; max, 39.4) kg equalled $29.5(5.7 ; 14.7 ; 46.5) \%$ of body mass. The average abdominal fat mass $\left(\mathrm{L}_{1}-\mathrm{L}_{4}\right)$ of $1.71(0.87 ; 0.45 ; 5.2) \mathrm{kg}$ accounted for $8.6(2.0 ; 4.3 ; 14.4) \%$ of total fat mass. The $\%$ fat mass located in the abdomen was related to \% body fat $(r=0.629)$ and body fat mass $(\mathrm{kg} ; r=0.68)$, accounting for 46 and $40 \%$ of the variance, respectively. Total fat mass accounted for $>85 \%$ of the variance in abdominal fat mass $(\mathrm{kg})(r=0.932)$, providing a linear estimate of abdominal fat mass approximating to $150 \mathrm{~g}$ per $\mathrm{kg}$ total fat mass (Figure 1).

In this cross-sectional comparison of women whose abdominal fat ranged between 4.3 and $14 \%$ of total fat mass, we found that the DXA method provided a reliable measurement of abdominal adiposity. The results of this study indicate that abdominal fat is relatively independent of the individual's \%body fat and is best predicted by total fat mass.

Further study will confirm whether a longitudinal change in abdominal fat occurs in accordance with the linear prediction derived from these data (i.e. $150 \mathrm{~g}$ loss of abdominal fat per $\mathrm{kg}$ loss of total body fat loss) following dietary intervention.

1. Freedman DS, Williamson DF, Croft JB et al. 1995. The National Health and Nutrition Examination Survey I (NHANES I): epidemiologic follow-up study. Am J Epidemiol 142, 53-63.

2. Faculty of Education and Health Sciences (2009) University of Limerick - Body Composition Study. http://www.ul.ie/bodycompositionstudy

3. Glickman SG, Marn CS, Supiano MA et al. (2004) J Appl Physiol 97, 509-514. 